\title{
Fascioliasis coledociana por Fasciola hepatica en cirugía de colecistitis crónica calculosa
}

\author{
Choledocal fasciolasis by Fasciola hepatica in surgery of chronic calculous cholecistitis
}

\author{
María Beltrán-Fabián ${ }^{1 a}$, Elena Muñoz-Zambrano ${ }^{1 \mathrm{~b}}$, Fabián Del Pozo-López ${ }^{2 a}$, \\ Silvia Gutiérrez-Cabezas ${ }^{2 b}$ \\ ${ }^{1 \mathrm{a}}$ Laboratorio de Enteroparásitos. Dirección Ejecutiva de Enfermedades Transmisibles. Centro Nacional de Salud Pública. Instituto Nacional de Salud. \\ ${ }^{1 b}$ Dirección Ejecutiva de Enfermedades No Transmisibles. Centro Nacional de Salud Pública. Instituto Nacional de salud. \\ $2^{\mathrm{a}}$ Laboratorio Central. Hospital Vitarte. \\ ${ }^{2 b}$ Medicina Interna. Hospital Vitarte.
}

\begin{abstract}
Resumen
Se reporta un caso de Fasciola hepatica en una mujer de 50 años de edad, natural de Huaral, procedente de un asentamiento humano del distrito de Ate en Lima, con un tiempo de enfermedad de siete dias caracterizado al inicio por dolor abdominal tipo cólico de curso progresivo en el epigastrio, dolor que se irradia a hipocondrio derecho. Luego presentó luego náuseas y vómitos en cuatro oportunidades, de contenido alimenticio y bilioso, por lo que se decide su hospitalización previa atención por emergencia. Al examen físico se encontró conjuntivas oculares levemente ictéricas, abdomen balonado, blando, depresible, no visceromegalia, Murphy positivo, ruidos hidroaéreos presentes, tórax y pulmones sin alteraciones. Posteriormente a los exámenes auxiliares, se diagnosticó colecistitis calculosa aguda y fascioliasis coledociana. En el acto quirúrgico se extrajo tres especímenes identificados como Fasciola hepatica. La paciente después de recibir el triclabendazol, concurrió al Hospital para la evaluación y control, habiendo logrado mejoria y bienestar.

Palabras clave: Fasciola hepatica, colecistitis crónica calculosa.
\end{abstract}

Abstract

We report a case of Fasciola hepatica in a 50 year-old woman born in Huaral, Lima, and living in Ate Vitarte, district of Lima, Peru, who was admitted because of seven days of disease characterized by progressive abdominal pain. She visited Ate's Hospital because of right hipocondrial and epigastric cramping abdominal pain in the morning accompanied by nausea and vomiting in four opportunities. Conjunctives were slightly icteric, abdomen was distended and depressible, there was no visceromegaly, Murphy's sign was positive, and thorax and lungs showed no alterations. At cholecistectomy three specimens identified as Fasciola hepatica were extracted. Following treatment with triclabendazole, clinical course was uneventful.

Key words: Fasciola hepatica, chronic calculous cholecistitis.

\section{An Fac med. 2011;72(2):141-5}

\section{INTRODUCCIÓN}

La fascioliasis es causada por Fasciola gigantica y Fasciola hepatica. Es un tremátode de cuerpo blando, similar a una hoja, conocido comúnmente como 'alicuya', 'duela', 'platija', 'pirihuín', 'saguaype', 'conchuela de hígado', 'distoma'. Este parásito es común en África, este de Europa, América Latina y América del Sur. Es prevalente en las zonas ganaderas y en zonas de nivel socioeconómico bajo. Jean de Brie, en 1379, se refirió a F. hepatica como el agente causal de la putrefacción del hígado. En 1551, Gesner demostró que la duela afectaba al ganado por el consumo de hierbas que crecen próximas al agua ${ }^{(1,2)}$.
Las metacercarias o formas infectantes de F. hepatica se encuentran en los caracoles Lymnaea, Fossaria, Galba y Pseudosuccinea, como hospederos intermediarios; estas abandonan al caracol y se adhieren a las frutas y vegetales de tallo corto y de consumo crudo en ensaladas, extractos (lechuga, berros, alfalfa, entre otros), siendo así la manera como se infecta el hombre. Los humanos eliminan los huevos con las heces y secreción biliar. Las formas adultas de F. hepatica son en su mayoría de localización hepática ${ }^{(3,4)}$. Debido a que la prevalencia en las áreas ganaderas es alta y va en incremento, se la considera como enfermedad emergente ${ }^{(5)}$. Las formas adultas son hermafroditas. Es característica la ramificación intensa de las gónadas, que abarca casi todo el cuerpo de la duela. Se encuentran mayormente en las vías biliares del hígado, en el ganado vacuno, ovino, porcino, equino, felino, roedores y el hombre, pudiendo haber otros de localización errática: pulmones, ganglios linfáticos, peritoneo, abdomen ${ }^{(6-8)}$. El tratamiento recomendado a todas las formas evolutivas, en ovinos infectados con fasciolas, es el 5-cloro-2-metiltio-6-(1-naftiloxi)ih-bencimidazol, ya que demostró una eficacia de $100 \%$ en todos los grupos tratados ${ }^{(9)}$.

Se describe el caso de una paciente con síndrome doloroso abdominal a 
quien inicialmente se le descartó una colecistitis aguda calculosa por los síntomas que presentó y que, finalmente, por anamnesis y exámenes complementarios, se determinó colecistitis crónica calculosa. La fascioliasis es una entidad a considerar en el diagnóstico diferencial con las colecistitis, sobre todo alitiásicas.

\section{CASO CLÍNICO}

Paciente mujer de 50 años de edad, natural de Huaral, domiciliada en el distrito de Ate Vitarte, en Lima, Perú. Acudió a Emergencia del Hospital de Vitarte con un tiempo de enfermedad de 7 días. Presentaba dolor abdominal tipo cólico en hipocondrio derecho y epigastrio, además de náuseas y vómitos de contenido alimenticio y bilioso, en cuatro oportunidades.

$\mathrm{Al}$ examen físico presentó conjuntivas oculares levemente ictéricas, abdomen balonado, blando, depresible, sin visceromegalia, doloroso a la palpación superficial y profunda en hipocondrio derecho, signo de Murphy positivo. En aparato cardiorrespiratorio, los ruidos cardiacos eran rítmicos, sin soplos, murmullo vesicular conservado en ambos campos pulmonares. Fue hospitalizada con diagnóstico clínico de colecistitis crónica reagudizada, por el antecedente de episodios similares de dolor abdominal similares al actual, durante los últimos meses.

En la ecografía abdominal se encontró hígado de $130 \mathrm{~mm}$ de longitud, sin prominencia del lóbulo izquierdo y sin lesión focal; la vesícula biliar distendida, de $105 \times 46 \mathrm{~mm}$, de paredes engrosadas de 4 a $5 \mathrm{~mm}$ en promedio, sin edema parietal, delimitándose múltiples imágenes litiásicas en su interior, de $6 \mathrm{~mm}$ de diámetro en promedio, algunas móviles y otras en región infundibular no móviles, sugestivas de estar impactadas (figura 1); colédoco dilatado, midió $11 \mathrm{~mm}$ de diámetro; las vías biliares intra y extrahepáticas se las observaba dilatadas, vena porta de 12 $\mathrm{mm}$ de diámetro a nivel biliohepático;

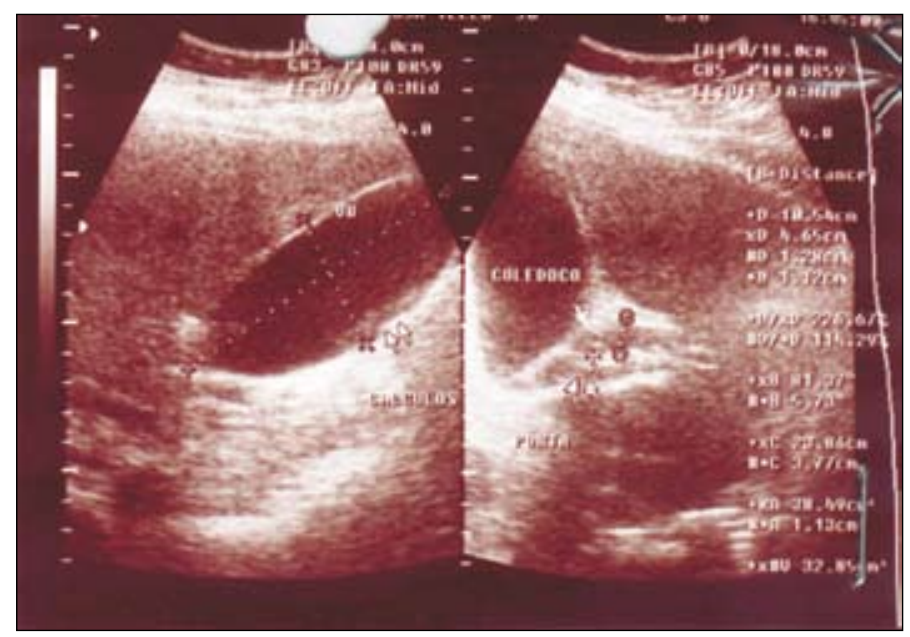

Figura 1. Ultrasonografía abdominal con imágenes de colestistis crónica y la vesícula biliar distendida por múltiples imágenes litiásicas.

bazo normal, de $95 \mathrm{~mm}$; páncreas de morfología habitual, medía $23 \mathrm{~mm}$, sin formaciones expansivas ni alteración en su estructura interna; planos grasos adyacentes conservados. Fue informada como colecistitis crónica calculosa, con signos de estar impactada, y dilatación de vías biliares intra y extrahepáticas.

Los resultados de laboratorio, en su primer día de hospitalización, fueron bilirrubina total $2,80 \mathrm{mg} / \mathrm{dL}$, bilirrubina directa $1,58 \mathrm{mg} / \mathrm{dL}$, bilirrubina indirecta $1,22 \mathrm{mg} / \mathrm{dL}$, fosfatasa alcalina $210 \mathrm{mg} / \mathrm{dL}$, TGO $90 \mathrm{UI} / \mathrm{mL}$, TGP $120 \mathrm{UI} / \mathrm{mL}$, hemoglobina $12,71 \mathrm{~g} / \mathrm{dL}$, hematocrito $40 \%$, leucocitos $10800 /$ $\mathrm{mL}$, abastonados $11 \%$ (desviación izquierda), segmentados $81 \%$, linfocitos $8 \%$, monocitos $0 \%$, basófilos $0 \%$, eosinófilos $0 \%$, plaquetas normales, glucosa $96 \mathrm{mg} / \mathrm{dL}$, urea $20 \mathrm{mg} / \mathrm{dL}$, creatinina $0,85 \mathrm{mg} / \mathrm{dL}$. Examen de orina con células epiteliales escasas, leucocitos 4 a 6/campo, hematíes 0 a 2/campo. Grupo sanguíneo A, Rh positivo, RPR y VIH no reactivos.

La paciente estuvo internada once días. Los primeros días de internamiento recibió por vía endovenosa ceftriaxona $1 \mathrm{~g} \mathrm{c} / 12$ horas y ranitidina

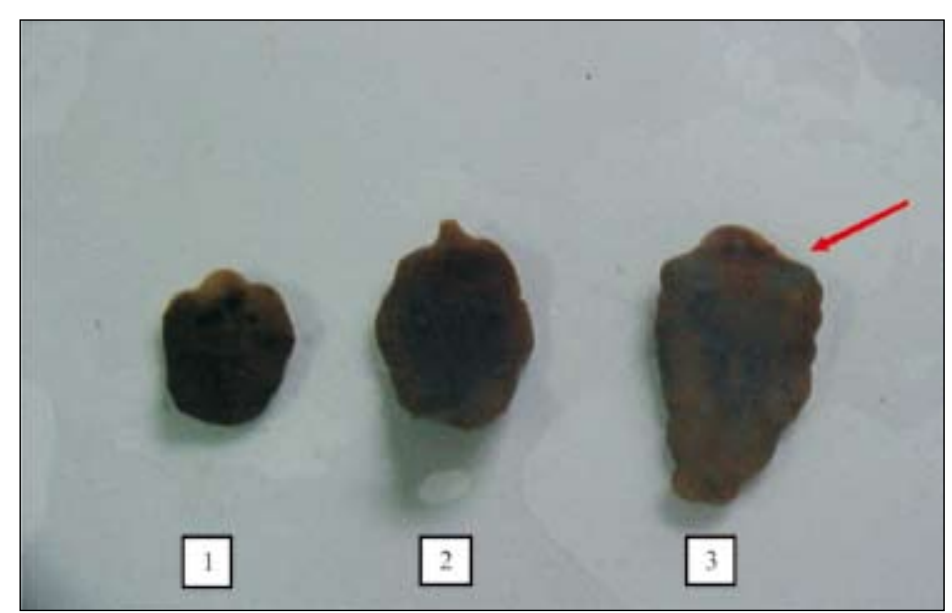

Figura 2. Fasciola hepatica: tres especímenes obtenidos del colédoco; el más grande midió $2,7 \mathrm{~cm}$. 




Figura 3. Fasciola hepatica $\mathrm{N}^{\circ} 1$ a $20 \mathrm{x}$. Forma juvenil sin colorear, mide $1,1 \times 0,7 \mathrm{~cm}$, después del aplanamiento surco oral subterminal.

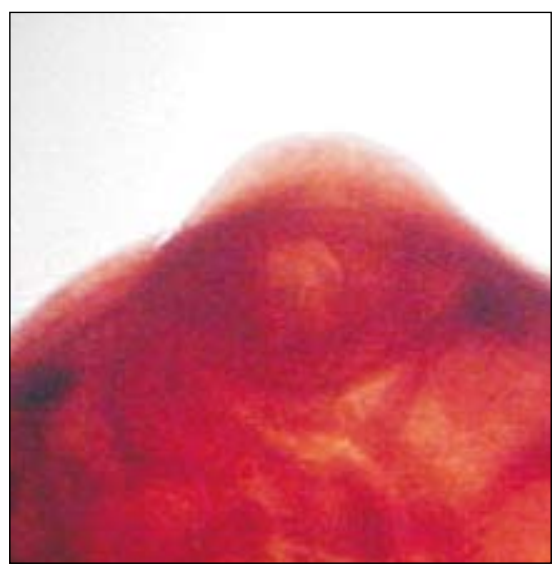

Figura 4. Fasciola hepatica $\mathrm{N}^{\circ} 1$. Extremo anterior coloreado con carmín clorhídrico. 100x.

$50 \mathrm{mg}$ cada ocho horas (en total por 9 días). Al tercer día postoperatorio, tuvo TGP $8 \mathrm{mg} / \mathrm{dL}$, TGO $6 \mathrm{mg} / \mathrm{dL}$, FA 205 $\mathrm{mg} / \mathrm{dL}$, bilirrubina total $1,2 \mathrm{mg} / \mathrm{dL}$, bilirrubina directa $0,7 \mathrm{mg} / \mathrm{dL}$, bilirrubina indirecta $0,5 \mathrm{mg} / \mathrm{dL}$, hemoglobina 12,7 $\mathrm{g} / \mathrm{dL}$, hematocrito $40 \%$, leucocitos 7 $900 / \mathrm{mL}$, abastonados $0 \%$, segmentados $65 \%$, linfocitos $34 \%$, monocitos $1 \%$, plaquetas normales.

Al cuarto día de internamiento fue operada, con el siguiente reporte operatorio: Hallazgos de vesícula distendida $+10 \times 6 \times 3 \mathrm{~cm}$, paredes engrosadas, conteniendo cálculos pequeños en poca cantidad y bilis color verde petróleo; conducto cístico de $3 \mathrm{~mm}$ de diámetro, colédoco $+10 \mathrm{~mm}$. Se realizó colecistectomía mixta, con exploración de vías biliares y se dejó drenes de Kehr y Penrose. El diagnóstico postoperatorio fue colecistitis aguda litiásica y fas-

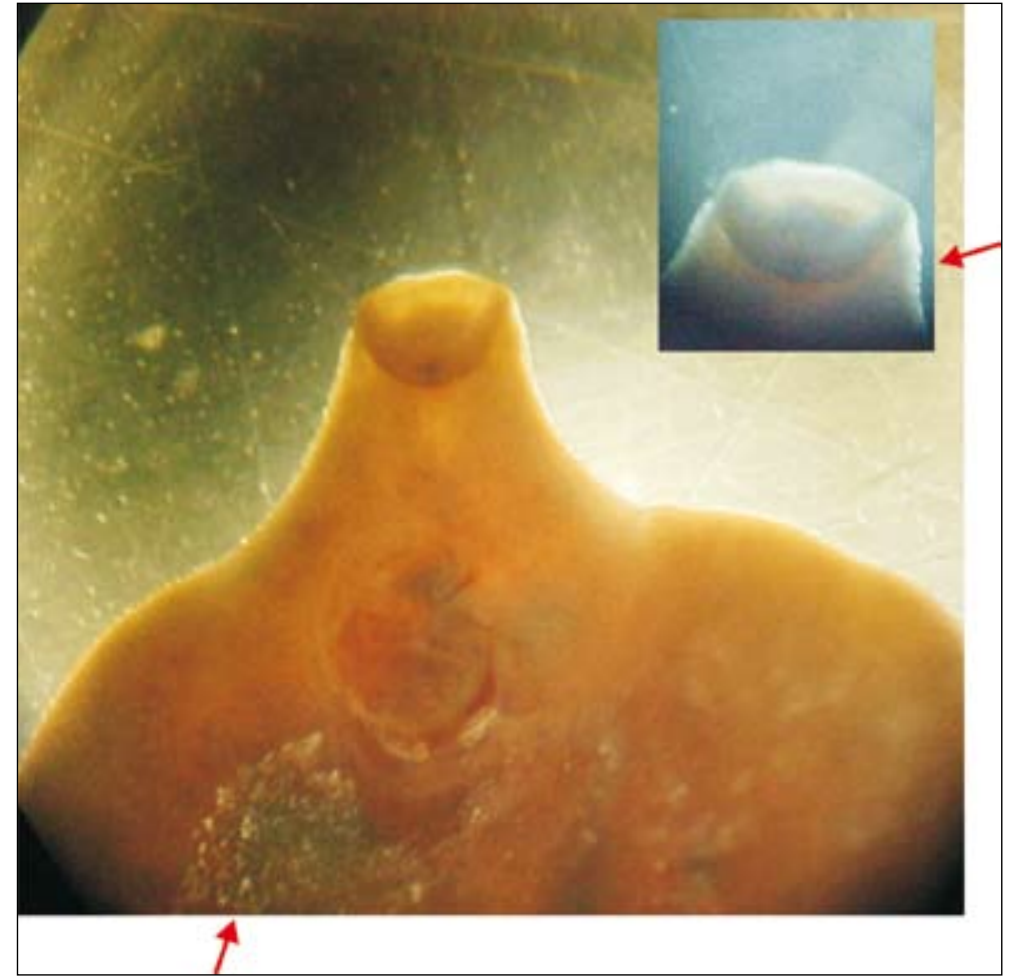

Figura 5. Fasciola hepatica $\mathrm{N}^{\circ} 2$ : mide $1,9 \times 0,7 \mathrm{~cm}$; se observa el surco oral en el extremo anterior y los huevos en el útero (flecha roja). 40x. En el ángulo superior derecho destacan las espinas. cioliasis coledociana, al extraerse tres especímenes identificados como Fasciola hepatica. La paciente fue referida al Hospital Cayetano Heredia, donde recibió tratamiento contra fascioliasis, con triclabendazol $10 \mathrm{mg} / \mathrm{kg}$ de peso.

Los tres especímenes identificados como Fasciola hepatica juvenil y adulto (figura 2), fueron fijados en formol al $10 \%$. En el laboratorio, estas duelas fueron lavadas con agua destilada, con repetidos cambios, para la eliminación del formol. Luego, se hizo el aplanamiento entre dos láminas, sujetas con pabilo por una semana, lo que permitió diferenciarlas mejor (figuras 3 a 6). Posteriormente, se realizó la coloración con carmín clorhídrico. Se muestra en la figura 4 las fuertes espinas curvas y dirigidas hacia atrás, antes de colorearlas, y las coloreadas con carmín clorhídrico (figuras 5 y 6 ).

\section{DISCUSIÓN}

Una variedad de gusanos de localización hepática, incluyendo Fasciola, pueden colonizar el árbol biliar, donde depositan sus huevos, formando cálculos biliares por masas o nudos de gusanos vivos o muertos; estos pueden causar obstrucción y algunas colangitis ${ }^{(9-11)}$.

La presencia de alicuyas o distomas se ve favorecida por el agua, donde habitan los caracoles como hospederos intermediarios. Si estos son invadidos por gran número de cercarias, los obliga a alimentarse en forma exagerada. El ganado, como hospedero definitivo, así como el hombre están con mayor riesgo de padecer de una distomatosis hepática, ocurriendo también la localización errática o ectópica ${ }^{(6,8,11-13)}$.

La infestación con Fasciola hepatica ocurre por el consumo de vegetales de 


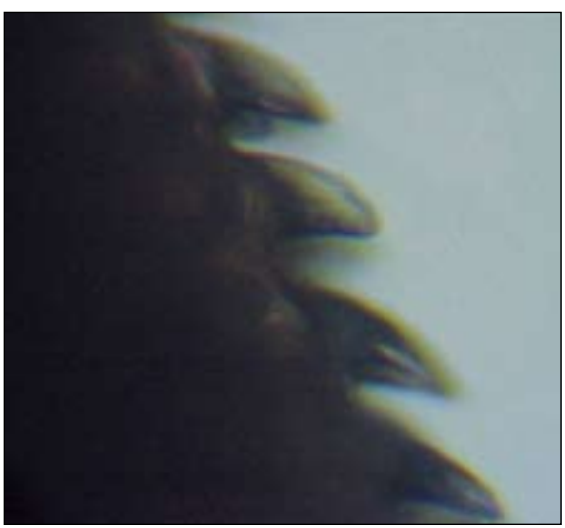

Figura 6. F. hepática, vista ventral; las espinas curvas se dirigen hacia atrás y son más gruesas. $400 X$.

tallo corto y consumo crudo o frutas; allí se establecen las metacercarias, que ingresan vía digestiva atravesando la pared del intestino. Después de 6 a 9 semanas (o de 42 a 60 días), las fasciolas jóvenes penetran la cápsula del hígado; algunas de ellas evolucionan hasta adultas en el árbol biliar ${ }^{(9,14)}$.

La Fasciola hepatica presenta 2 estadios. La fase aguda e invasiva coincide con la invasión hepática; el paciente puede tener procesos de fiebre, dolor del cuadrante abdominal derecho, eosinofilia, hepatomegalia y diversas manifestaciones gastrointestinales ${ }^{(10,14-16)}$; y la fase crónica es cuando los adultos de Fasciola están en el árbol biliar, donde

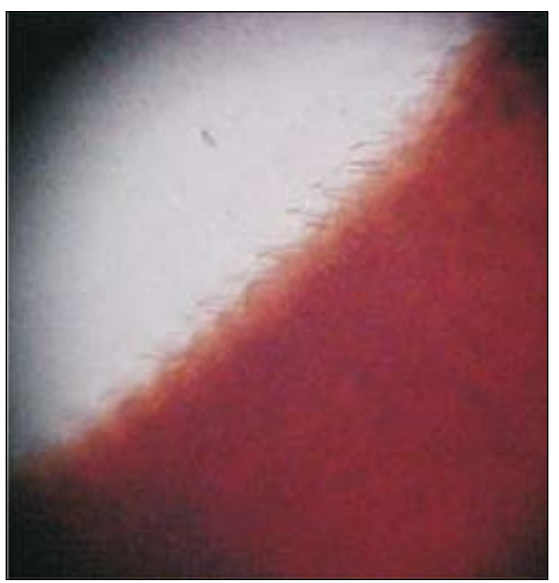

Figura 8. Espinas de Fasciola coloreadas con carmín clorhídrico 100X.

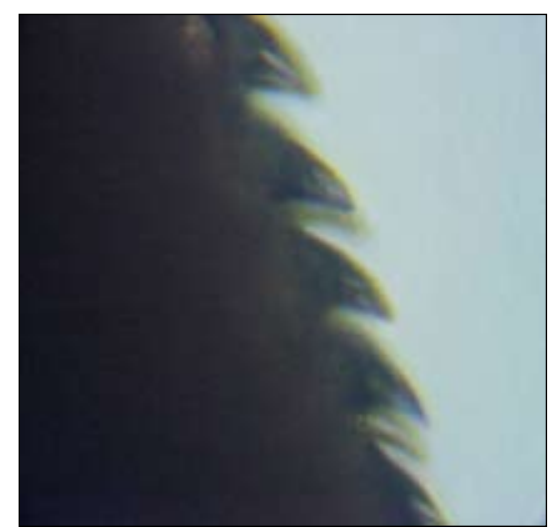

Figura 7. F. hepática, vista dorsal; se observa las espinas antes de ser coloreadas. 400X.

eliminan huevos que pueden ser encontrados en las heces de los pacientes infestados; los huevos dan lugar a los miracidios, que son buscados por el caracol; en 5 a 6 semanas, genera el esporoquiste y produce partenogénesis 5 a 6 redias; estas, en condiciones desfavorables, dan origen a redias hijas y nietas, las que en condiciones apropiadas dan cercarias y luego metacercarias, que pueden resistir hasta 12 meses en humedad ${ }^{(18)}$. La fascioliasis es considerada como una enfermedad emergente y reemergente, por la gran capacidad de expansión del agente causal y los hospederos intermediarios, considerados también como vectores ${ }^{(19)}$.

En la fase aguda, el diagnóstico de fascioliasis es por la detección de anticuerpos anti Fasciola, mediante aplicación de los métodos doble difusión arco 2 (DD2), ELISA e inmunoblot.

En la fase crónica, se diagnostica, en el análisis cualitativo o semicualitativo, por la observación de huevos en la muestra fecal, contenido duodenal o secreción biliar, dado por el sistema de cruces por campo microscópico por cm: 2 a 5 huevos/cm $1+, 6$ a 10 huevos/cm $2++$, y más de 10 huevos $/ \mathrm{cm}$ $3+++{ }^{(17)}$. En el análisis cuantitativo, por aplicación del método de Kato Katz se obtiene el número de huevos por gramo de heces (hgh), determinando una infección leve (1 a 99 hgh), moderada (100 a 299 hgh) y severa (mayor de 300 hgh) ${ }^{(16,17)}$. Desde el punto de vista práctico y la experiencia en estos diagnósticos, nos permite referir que hay una relación entre infecciones con $3+++$, $2++$ y $1+$ con infección severa, moderada y leve, respectivamente.

En el presente estudio se hizo el diagnóstico por la diferenciación de los especímenes juvenil y adultos. Es importante diferenciar la forma juvenil, así como su preservación después de la obtención de los distomas del hospedero y hacer el diagnóstico diferencial con Echinostoma ${ }^{(8)}$. Se observó de manera nítida las espinas en los bordes del espécimen (figuras 5 a 8).

En otros trabajos, como el realizado por López y col. ${ }^{(20)}$, utilizaron la tomografía computarizada, electrosonido y resonancia magnética, en los cuales se puede ver lesiones focales hipoecogénicas, anfractuosas, bien definidas, de tamaño variable, con tendencia a coalescencia ${ }^{(21)}$, en $96 \%$ de los casos ${ }^{(22,23)}$, aunque también se describe ecogenicidad variable ${ }^{(24)}$.

En el presente trabajo se utilizó la ultrasonografía abdominal y la confirmación determinante por diagnóstico parasitológico, lavado, aplanamiento y coloración con carmín clorhídrico de los distomas. Se trató de un caso de fascioliasis coledociana por Fasciola hepatica y colecistitis crónica calculosa. Se muestra fotomicrografías que hacen patente espinas fuertes dirigidas hacia atrás en toda la cutícula, más visibles en el extremo anterior (Figura 4 y 5).

\section{REFERENCIAS BIBLIOGRÁFICAS}

1. Mehlorn H, Piekarski G. Fundamentos de Parasitología: Parásitos del hombre y de los animales domésticos. Trad. O Torres Quevedo. 3 ed. Zaragoza, España: Acribia. 1993:391.

2. Miyazaki I. Helminthic Zoonoses. International Medical Foundation of Japan Tokyo, 1991:494 pp.

3. Lam R, Flores VM, Terashima A, Samalvides F, Miranda $\mathrm{E}$, Tantatalean $\mathrm{M}$, et al. Hiperendemicidad de fasciolosis humana en el Valle del Mantaro: factores de riesgo de la infección por Fasciola hepatica. Rev Gastroenterol Peru. 2004;24:158-64.

4. Figueroa JA, Ramírez A, Quiroz H. Especialidad Médica. Fasciolosis F.M.V.Z.U.N.A. Disponible en www.Fmvz.UNAM.mx/mxpex/Veter_Mex/aspectos/ Fasciola/acu News 05. 
5. Fiel NA. Enfermedades parasitarias de importancia económica en bovino; bases epidemiológicas para la prevención y control. Buenos Aires, Argentina: Hemisferio Sur. 1988.

6. Beltrán $\mathrm{M}$, Tantaleán $\mathrm{M}$, Meza $\mathrm{H}$, Lozano M. Fasciolosis hepática de localización errática. Rev peru med exp salud publica. 2004;21(4):277-9.

7. Aghajanzadeh M, Sarshad A, Ebrahimian R. Pneumothorax a rarity in fascioliasis. Arch Iranian Med. 1999;2:213.

8. Beltrán M. Primer reporte de infección humana por Echinostoma sp en el Perú. Rev med exp salud publica. 2002;19 Supl:S24.

9. Loja Oropeza D, Alvizuri Escobedo J, Vilca Vásquez M, Avilés R, Sánchez M. Hematoma hepático subcapsular por fasciola. Rev. gastroenterol. Perú. 2003;23(2):142-8.

10. Dobrucali A, Yigitbasi R, Erzin Y, Sunamak O, Polat E, Yakar H. Fasciola hepatica infestation as a are cause of extrahepatic cholestasis. World J Gastroenterol. 2004;10(20):3076-7.

11. Maco V, Marcos L, Montenegro J, Bellido J, Terashima A, Gotuzzo E. Un caso de obstrucción de dren de Kehr por Fasciola hepatica en una paciente post colecistectomizada por colangitis aguda. Reporte de caso y revisión de la literatura. Parasitol Latinoamer. 2003;58:152-8.

12. Roses LL, Alonso D, Iñique F, Mateo A, Bal M. Agüero J. Hepatic fasciolasis of long term evolution. Diagnosis by E.R.C.P. Am J Gastroenterol. 1993;88:2118-9.
13. Beltrán M, Muñoz ME, del Pozo F, Barberena F, Gutierrez S. Fasciolosis hepática coledociana en colecistitis calculosa. Rev Peruana Parasitología. 2008;17:54.

14. Blancas G, Teraschima A, Maguiña C, Vera L, Alvarez $\mathrm{H}$, Tello R. Fasciolosis humana y compromiso gastrointestinal: Estudio de 277 pacientes en el Hospital Nacional Cayetano Heredia. 1970-2002. Rev Gastroenterol Perú. 2004;24:143-57.

15. Quiroz H, Figueroa JA. Estudios de fasciolosis en México, de 1879-2006. México DF: Editores: Héctor Quiroz Romero y Juan Antonio Figueroa Castillo. 2010:1708-1.

16. Mas-Coma S. (2004). Human fascioliasis. En: Crotruvo JA, Dufour A, Rees G, Bartram J, Carr R, Cliver DO, et al. eds. World Health Organization (WHO), Waterborne Zoonoses: Identification, Causes and Control. London, UK: IWA Publishing. 2004:30522.

17. Beltrán M, Tello R, Naquira C. Manual de procedimientos de Laboratorio para el diagnóstico de los parásitos intestinales del hombre. Serie de Normas Técnicas Nº 37, Lima, 2003.

18. Mas-Coma S, Bargues MA, Mas Coma VS. Fascioliasis and other plant-borne trematode zoonoses. Int J Parasitol. 2005;35:1255-78.

19. Fredes F. Fascioliasis humana y animal. Mon Electr patol Vet. 2004;1:38-67.

20. López A, Silva C, Busel D. Fasciolasis hepática: Reporte de un caso y revisión de la literatura. Rev chil radiol. 2004;10(3):118-23.
21. Bassily S, Iskander M, Youssef FG, El-Masry N, Bawden M. Sonography in diagnosis of fasciolasis. Lancet. 1989;1(8649):1271-972.

22. Kabaalioglu A, Cubuk M, Senol U, Cevikol C, Karaali K, et al. Fasciolasis: US, CT and MRI findings with new observations. Abdom Imaging. 2000;25:400-4.

23. Cosme A, Ojeda E, Poch M, Bujanda L, Castiella A, Fernández J. Sonographic findings of hepatic lesions in human fascioliasis. J Clin Ultrasound. 2003;31:358-63.

24. Van Beers B, Pringot J, Geubel A, Trigaux JP, Bigaignon $\mathrm{G}$, et al. Hepatobiliary fasciolasis: Noninvasive imaging findings. Radiology. 1990;174:809-10.

Trabajo recibido el 9 de julio de 2010 y aceptado para publicación el 12 de marzo de 2011.

\section{Correspondencia:}

María Beltrán-Fabián

Laboratorio de Enteroparásitos. Dirección Ejecutiva de Enfermedades Transmisibles. Centro Nacional de Salud Pública. Instituto Nacional de Salud.

Correo electrónico:

mbeltran@ins.gob.pe

mariabeltranf@hotmail.com 